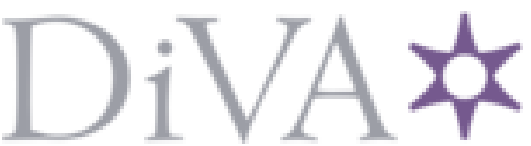

http://www.diva-portal.org

Postprint

This is the accepted version of a paper presented at 1st International Workshop on Machine Learning for Smart Wireless Networks.

Citation for the original published paper:

Finne, N., Eriksson, J., Voigt, T., Suciu, G., Sachian, M-A. et al. (2021)

Multi-Trace: Multi-level Data Trace Generationwith the Cooja Simulator

In:

N.B. When citing this work, cite the original published paper.

Permanent link to this version:

http://urn.kb.se/resolve?urn=urn:nbn:se:uu:diva-459163 


\title{
Multi-Trace: Multi-level Data Trace Generation with the Cooja Simulator
}

\author{
Niclas Finne $^{1}$, Joakim Eriksson ${ }^{1}$, Thiemo Voigt ${ }^{1,2}$, George Suciu ${ }^{3}$, Mari-Anais Sachian ${ }^{3}$, \\ JeongGil Ko ${ }^{4}$, Hossein Keipour ${ }^{1,5}$ \\ ${ }^{1}$ Research Institutes of Sweden (RISE), Sweden, ${ }^{2}$ Uppsala University, Sweden \\ ${ }^{3}$ BEIA, Research Department, Romania, ${ }^{4}$ Yonsei University, South Korea, \\ ${ }^{5}$ Blekinge Institute of Technology, Sweden
}

\begin{abstract}
Wireless low-power, multi-hop networks are exposed to numerous attacks also due to their resource-constraints. While there has been a lot of work on intrusion detection systems for such networks, most of these studies have considered only a few topologies, scenarios and attacks. One of the reasons for this shortcoming is the lack of sufficient data traces that are required to train many machine learning algorithms. In contrast to other wireless networks, multi-hop networks do not contain one entity that can capture all the traffic which makes it more difficult to acquire such traces. In this paper we present MultiTrace. Multi-Trace extends the Cooja simulator with multi-level tracing facilities that enable data logging at different levels while maintaining a global time. We discuss the opportunities that traces generated by Multi-Trace enable for researchers interested in input for their machine learning algorithms. We present experiments that show the efficiency with which Multi-Trace generates traces. We expect Multi-Trace to be a useful tool for the research community.
\end{abstract}

\section{INTRODUCTION}

Low-power wireless multi-hop networks such as wireless sensor networks and many Internet of Things systems are promising applications in domains of high industrial and societal relevance such as healthcare, agriculture, aviation and aerospace, civil infrastructure monitoring and process control in industrial settings. They offer, however, also new attack vectors mainly because of their constraints in memory, computing power and energy and the wireless nature of the communication [1]-[3]. These new attack vectors include, for example, denial of service attacks through jamming that aim to degrade network performance, by depleting devices' batteries by causing additional packet loss and delays.

To defend against attacks, solutions often have three main components [4]: First used are components to prevent attacks. Second are components to detect an attack and third, components to mitigate an attack. If the first line of defense, the attack prevention component, has been crossed, then the second line of defense aims to detect any suspicious behaviour. The latter is done via intrusion detection systems (IDS). Due to resourceconstraints of low-power embedded platforms, designing efficient intrusion detection systems is extremely challenging [4]. Hence, there has been a flood of research papers proposed on this topic. For example, one of the earliest papers on intrusion detection by Raza et al. [5] has been cited more than 650 times. Intrusion detection systems often make use of various machine

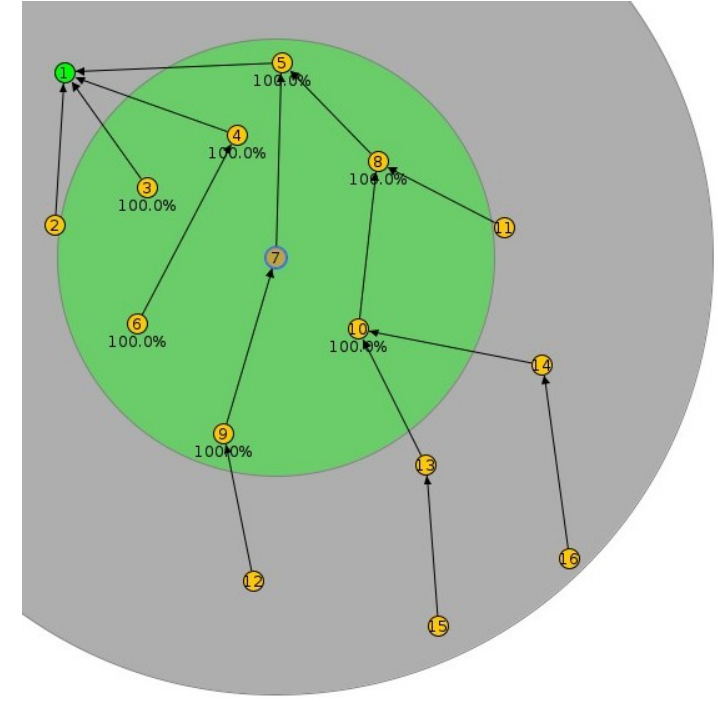

Fig. 1. In wireless multi-hop networks, there is no entity that can overhear all packets.

learning techniques [6], [7]. Nevertheless, to be effective in various attack scenarios, machine learning-based algorithms require a lot of training data.

In many research domains, there exists quite a number of data traces that include, for example, packet traces such as the NSL-KDD dataset [8]. In the area of low-power wireless multi-hop networks, despite the significant body of research, we were not able to identify similar data sets. Instead, researchers use their own, often limited data sets for algorithm design and validation. In contrast to many other network architectures, sensor networks hold a rather unique architecture in which a gateway node is often designed to overhear packets from a large subset of individual nodes operating in its subnet (c.f., Figure 1 where the gateway is depicted as the green node and the green and gray regions represent the communication range and interference range of a sensor node).

In this paper, we extend Cooja [9], a widely used simulation tool for multi-hop wireless networks to generate data traces that can be used to train machine learning algorithms for intrusion detection in low-power resource-limited networks.

Contributions. We make the following contributions in this 
paper. After presenting background on the Cooja simulator, in Section III we present our multi-level trace generation facilities that consist of logs at different levels with some additional scripts to define simulation scenarios. As all logs are created in the same simulation, they share a global timestamp which allows the merging and combining of different logs in a userdefined manner.

Section IV discusses the opportunities the traces generated by Multi-Trace enable for researchers interested in creating input traces for their machine learning algorithms. For example, our tracing facilities enable users to derive which information is available at which entity in the network at any time instance. The next section briefly discusses Multi-Trace's implementation. In contrast to other efforts to construct traces with Cooja [10], we modify Cooja itself to support efficient trace generation. Section VI evaluates some interesting aspects of Multi-Trace to demonstrate the usability of the tool. Our results show that we can generate more than 160 traces per hour when we have 20 nodes in the simulation. We expect Multi-Trace to be a useful tool for the community that allows users to generate data traces for their specific purposes. This way, we expect Multi-Trace to help to push forward the field of security for low-power wireless multi-hop networks.

\section{THe COOJA SIMUlator}

The Cooja simulator [9] has originally been designed to simulate networks of resource-constraint networked embedded devices running the Contiki operating system [11]. Nevertheless, its flexibility allows the use of binaries designed in other operating systems as well [12], [13].

Specifically, Cooja can execute Contiki programs on different levels. Cooja can run the program code as compiled native code directly on the host CPU, or it can run compiled program code in MSPSim. MSPSim is a Java-based instruction level emulator of the MSP430 microprocessor series [14]. Both methods allow the simulation of directly-deployable code, i.e., code that can run on hardware without any modification. In addition, Cooja is able to simulate nodes developed in Java at the application level. The disadvantage of this approach is that these nodes are not deployable binary; thus, they can only validate the operations and not the actual deployable code itself. Emulating nodes with MSPSim inside Cooja allows control and retrieval of more fine-grained execution details compared to Java-based nodes or nodes running native code. Native code simulations are more efficient than node emulations. Hence, for the purpose of generating traces for machine learning algorithms, native code simulations are the best option. Cooja also comes with several built-in tools. One is the so-called radio logger, which captures all packets that are transmitted by nodes in the simulation with a universal timestamp.

Contiki [11] and its newer version Contiki-NG are operating systems for resource-constrained networked embedded systems such as sensors and actuators. They implement a number of network protocols for low-power communication with a focus on recent standards such as the Routing Protocol for

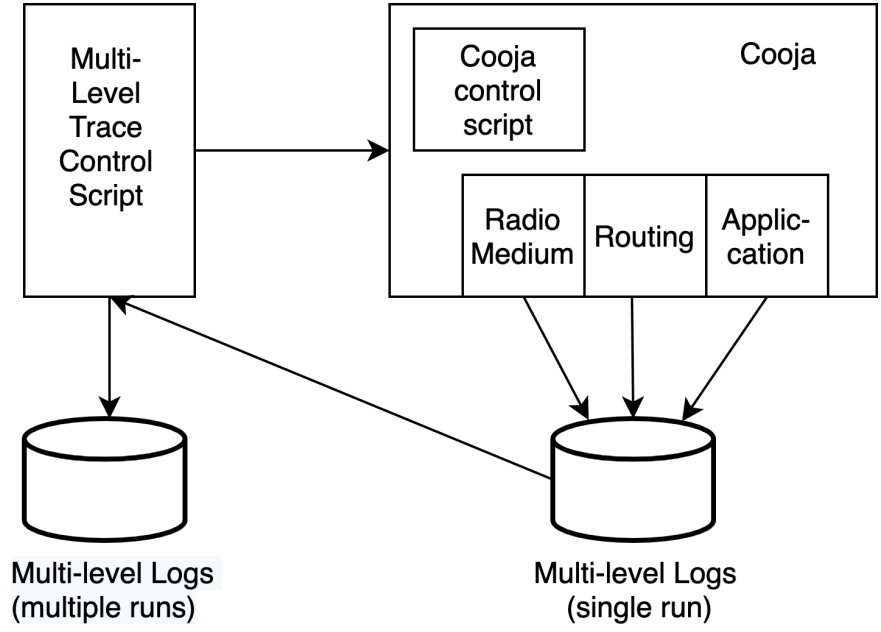

Fig. 2. Multi-Trace extends Cooja to generate multi-level logs.

Low-Power and Lossy Networks (RPL) [15] and TSCH [16]. Contiki also offers on-line power profiling mechanisms [17], [18] that estimate the energy consumption by measuring the duration each component operates under various modes such as low-power mode, packet transmitting or receiving. Logging the output of these tools is useful for detecting attacks that impact the nodes' energy consumption [10].

\section{iII. Multi-level Trace Generation}

The goal of this work is to generate a set of detailed and generic data traces that can be used as training data for various machine learning algorithms. Low-power networks typically consist of both resource-constrained nodes and one or more less resource-constrained gateways. Therefore, machine learning algorithms can be designed to run on any of these heterogeneous platforms or in some cases exploit both by splitting the operations. Indeed, there exists quite some research on splitting (or offloading) deep learning pipelines between resource-constrained nodes and more capable entities [19].

\section{A. Multi-level Logging}

We provide four different types of data we are able to extract from Cooja simulations: First, application developers can print data specific to their application using standard Contiki log messages. At this level, users have the full freedom to customize their log messages. Second, Cooja has a radio logger plugin that logs all data traffic. This data is available in the pcap format, a format originally used by the pcap API of tcpdump [20]. Third, we enable the logging of radio transmissions from the radio medium. While the radio logger plugin logs all messages that are in the air, it does not include information about which nodes received a certain radio transmission. In particular when broadcast messages are sent in multi-hop networks, it is difficult to derive which nodes received a message based only on the radio logger. This information could be added at the application layer, but this would require additional effort by the programmer and could lead to convoluted code. Using information from the radio 
medium instead gives a clear log of which nodes received or were interfered by each radio transmission. Fourth, there is an event $\log$ for events during the simulation. For example, a simulation can $\log$ an event when the network has reached a steady state to make it easier to ignore the startup phase of a simulation. Or when an attack is started or stopped to indicate during which times an attack is active. Note that while we offer opportunities for logging at different levels, they all share the global simulation time. This facilitates the fusion of the information from the different logs.

\section{B. Scenario Setup}

Many machine learning algorithms require a lot of data traces. For at least supervised algorithms for intrusion detection, a single scenario is either a trace from a run with or without an attack. We hence need to perform many simulation runs, simulating different scenarios even for one single attack. Such scenarios can differ in many aspects including the number of nodes, the physical or logical distribution of the nodes, the traffic pattern and load, the node that executes the attack as well as the application running on the nodes. Our trace generation facility therefore includes a Python script that based on a simulation, generates new simulations with other properties such as new randomized topology. We also provide a script that runs Cooja on a set of simulations in order to generate a data trace for each simulation. This script sets up each simulation in Cooja and executes the simulation to its end.

For many attacks on multi-hop networks, executing an attack before the network is connected and stable is not of interest. Therefore, an execution that contains an attack, makes sure that the attack is not started before the network is fully setup. We consider the network as fully set up when the gateway has received at least one message from all nodes in the network.

\section{Attack Implementation}

When generating data traces, we typically do not want an attack to be active for the full simulation time. Instead, an ideal data trace would be captured by configuring a properly operating network (from initialization to stabilization) prior to the introduction of an attack. There are multiple approaches to achieve this. The simulation can dynamically add and remove nodes specially coded to attack or the simulation can instruct the nodes when to start or end an attack. We have selected the latter approach by implementing the attack as a module linked with all nodes and added a command line interface (CLI) to the nodes. The CLI is used to let the simulation run commands on the nodes via each node's serial port; thus, to start or stop an attack, the simulation simply sends an "attack" command over the serial port. This also means that the same node firmware can be used on real hardware, for example, to perform the same experiments on sensor network testbeds.

There are a number of possible attacks that have been identified in RPL/6LoWPAN networks [1] but to showcase the effectiveness of Multi-Trace we select the Blackhole attack.
In the Blackhole attach, a node is participating normally in a network and at some time it starts a denial of service (DoS) attack by broadcasting fake routing information to trigger its neighbors to switch to the attacking node as their routing parent. The attacking node's own routing parent will also hear the fake routing information, but the attacking node will ignore any route request from its routing parent; thus, the routing will not immediately switch to route via the attacking node. This allows the attacking node to selectively drop data from its routing children while the attacking node itself still is part of the network and can deliver its own data as normal for some time after start of the attack.

The routing is handled by the RPL implementation in the Contiki-NG core, but we make no modifications of the core OS for the attack implementation. Contiki-NG provides a mechanism to add a hook into the network stack that will be called whenever an IP packet is sent or received. This hook can be used not only to inspect and modify IP packets but also to discard IP packets. The attack module exploits this hook to catch outgoing DIO packets (used in RPL to advertise routes) and modify them to include a superior, fake routing rank. The hook is also used to catch incoming DIO packets and only allow DIO packets from its routing parent to reach the network stack to prevent the attacking node's own routing implementation from being affected by the attack. Furthermore, the hook is used to catch incoming DAO packets (used in RPL to request routes) and prevent them from reaching the network stack at the same time as a fake DAO ACK with an unconditional accept is sent as reply to any node except the attacking node's own routing parent. This will let any node that requests a route to immediately switch and use the attacking node as routing parent. Finally, the hook is also used to discard any outgoing IP packets not originated from the attacking node: essentially dropping all forwarded IP packets to or from its children.

\section{OPPORTUNITIES}

Our multi-level tracing facilities offer multiple opportunities for the evaluation of machine learning algorithms. We believe that some of them have not been considered in depth by the research community. Where in the network machine learning algorithms can be executed depends on the knowledge of the involved entities, i.e., the nodes and the gateway. Besides the knowledge, also the available resources that are typically much lower on the nodes compared to the gateway, must be considered. When deploying machine learning algorithms it is necessary to understand where the knowledge is available. For example, the gateway can collect information from the nodes in a network but only if the communication reaches the gateway.

\section{A. Packet Sniffers}

In multi-hop networks, a packet that is being transmitted can only be overheard by nodes in the vicinity. Even the gateway is not able to hear every packet that is in the air. If one wants to capture all the traffic, there is a need to set up 
multiple packet sniffers [21] that must be strategically placed in order to be able to overhear all traffic. This is in contrast to single-hop networks as, for example, WiFi 802.11 where one sniffer is sufficient [22]. Moreover, many network and MAC protocols [23] make use of multiple frequencies which implies that packet sniffers operating on multiple frequencies are required. The data that is available to packet sniffers is similar to the data captured by the radio logger and hence available in Multi-Trace.

\section{B. Node-local Knowledge}

Communication is typically the most energy-expensive tasks nodes in multi-hop sensor networks perform and requires more energy than sensing or computing. Hence, it is in many scenarios desirable to execute machine learning algorithms locally on the nodes rather than transmitting data to the gateway or a cloud [24] [25] [26]. Node-local knowledge can be traced either by application level information or by the information that nodes transmit to other nodes which is available from the radio logger.

\section{Gateway and Distributed Knowledge}

As discussed above, the gateway is typically the least resource-constrained device in a network of embedded devices. In addition, it is typically connected to a more powerful edge or the cloud. Hence, it is able to execute more demanding machine learning tasks than the other nodes in the network.

In general, one cannot assume that the gateway has global knowledge about the states of all nodes and the whole network. Since communication is energy expensive, it is impossible that nodes send all their status information to the gateway. In this context, Multi-Trace's tracing facilities enable users to explore, for example, the most important parameters that the gateway should have available to detect certain attacks. In addition, it allows to estimate the energy cost for sending network monitoring information to the gateway.

Furthermore, Multi-Trace enables users to design and evaluate attack detection algorithms where groups of nodes collaborate to detect attacks. These distributed algorithms may or may not involve the gateway. Note that in such scenarios, it is important to not only understand which messages have been transmitted (which can be deducted from the radio logger) but also which packets nodes have received.

\section{IMPLEMENTATION}

To make it easier to collect simulation logs, we have extended Cooja with a centralized log mechanism that saves simulation data to a set of log files in a folder. The folder name is named based on the simulation plus a simulation id to ensure each run of a simulation will result in a new set of data traces. The data traces can optionally be auto compressed to reduce disk usage. This makes it easier to script Cooja to run without GUI for multiple simulation files in sequence without requiring any user interaction. In addition to the normal pcap file with radio packet data, a more detailed radio $\log$ has been added based on information from the simulation radio medium. This

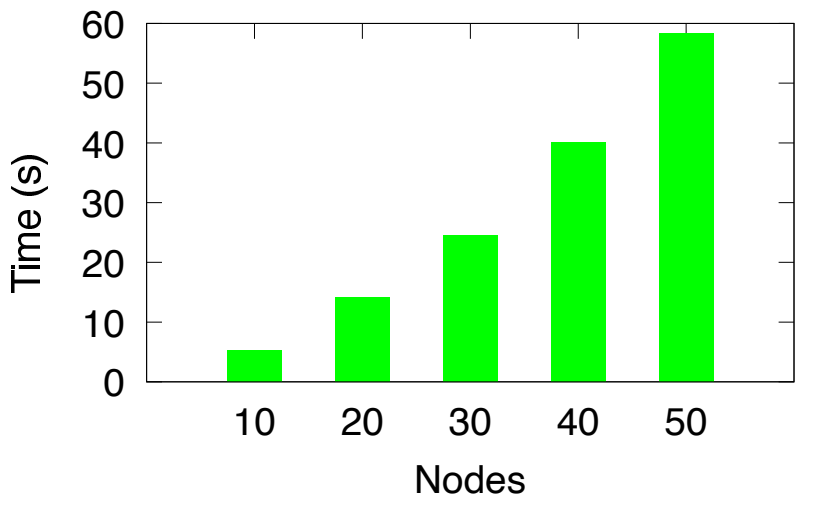

Fig. 3. Simulation times for varying numbers of nodes.

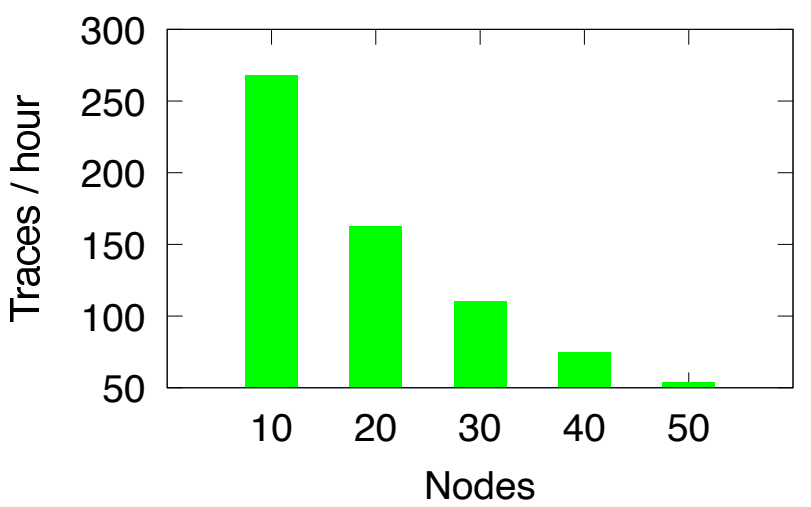

Fig. 4. Generated data traces per hour for varying numbers of nodes.

radio log includes among other things the radio channel, start and end time of transmission, and information about which nodes receive or are interfered by each radio transmission. The normal pcap file only contains radio packet data without any information about which nodes are in range to receive the radio transmissions.

In addition to the Cooja changes, we have developed a set of Python scripts to generate simulations, execute sets of simulations, and parse simulation results. These scripts can from a set of simulations generate any number of new simulations with randomized topologies and other modifications to provide a new set of simulations with varying conditions. The simulations can be set up with or without attacks from start or by using the Cooja ScriptEngine to dynamically start or stop attacks during the simulation. The Cooja ScriptEngine executes JavaScript code included in the simulation during execution of the simulation. This JavaScript code has access to the full Cooja API and thereby full control of the simulation. Typically, the ScriptEngine is used to determine when the network has reached a steady state after simulation start, start or stop network attacks, and also to decide when a simulation should end. 


\section{Evaluation}

We have selected a data collection application as case study where the nodes periodically report data to a sink node via a multi-hop network. The simulations include a control script that waits until the sink has received at least one radio packet from each client node. The script then logs an event that the network has reached a steady state and no longer is in the startup phase. If the simulation should include an attack, this is also when the control script starts an attack by sending a command to a predetermined or random node. The simulation then executes for one simulated hour before it stops. In our tests of this scenario one simulated hour is enough time for the network to stabilize after the start of an attack. Each such state change in the simulation is logged as an event in order to make it easy to ignore the initial network setup phase in the data traces. Any start or stop of network attacks is also logged as an event with a timestamp.

As mentioned earlier in Section II, nodes can be executed on different levels in Cooja. Emulating hardware gives cycle accurate timing but is much slower to execute. Here we have selected to use native motes when generating data traces. Executing nodes compiled to native code is much faster.

Figure 3 shows the execution times in Cooja to run one simulation to its end and generate one data trace for a set of simulations with varying numbers of simulated nodes. The tests have been executed on a MacBook Pro with $2.9 \mathrm{GHz}$ Intel Core $\mathrm{i} 7$ from 2016. The figure shows only the time to execute a simulation and how it increases with the number of emulated nodes. Figure 4 shows the number of traces that can be generated per hour using the same simulations as in Figure 3 but taking into account the full setup time of starting a Java VM for Cooja, load the simulation, build the node source code and run the simulation to its ends.

These results demonstrate that Multi-Trace is able to generate traces fast enough to make it a useful tool for the generation of data traces for machine learning input. The data trace generation will be faster on a more powerful machine. As Cooja currently only uses a single CPU core, it would also be possible to further increase the speed by running multiple simulations in parallel.

\section{RELATED WORK}

There are quite some studies that use Cooja to generate training sets for intrusion detection systems. Sharma et al. intend to detect several known attacks using machine learning analysis [27]. They create 300 traces using Cooja. Ponge et al. also use Cooja, but their evaluation uses just a few topologies [28]. The same seems true also for other efforts including the ones by Napiah et al. [29] and by Al-Hadhrami and Hussain [30]. Multi-Trace enables users to create a large number of topologies. Verma and Ranga created a dataset they call RPL-NIDDS17 [31] using another simulator to perform intrusion detection using ensemble learning [32]. It seems that this data set is no longer available.

None of the authors have gathered traces that provide enough input for, for example, deep neural networks. One major reason might be the lack of support that Multi-Trace provides. Wang et al. have shown that deep neural networks are a good option when handling time-series data and that they are feasible at the edge, i.e., in a powerful gateway [33].

Essop et al. [10] and Kfoury et al. [34] have presented studies in which they also generate data traces using the Cooja simulator. They capture the messages from the existing radio logger and from power trace whereas we enhance Cooja itself to facilitate the generation of trace files.

There are data sets in the broader IoT community not only focusing on low-power multi-hop networks. Anagnostopoulos et al. provide data from a smart home setup where they collect traces from IoT devices running different type of network protocols [35]. Suciu et al. aim to detect threats on IoT systems for healthcare using traces from building management systems running the BACnet protocol [36].

\section{CONCLUSIONS}

Intrusion detection systems based on machine learning typically rely on a large amount of training data. In this paper we present Multi-Trace, an extension of the widely used Cooja simulator that facilitates the generation of data traces for lowpower multi-hop networks. These traces consist of logs at different levels that share a global timestamp enabling users to merge and combine the different logs. We show that MultiTrace's flexibility can support the generation of training data for different approaches to intrusion detection systems. We demonstrate that Multi-Trace can generate traces at high speed which makes it a valuable tool for the research community ${ }^{1}$.

\section{ACKNOWLEDGEMENTS}

This work was supported by the ITEA 3 project STACK funded by the Swedish Innovation Agency VINNOVA and by the Korean Ministry of Trade, Industry and Energy/Korea Institute for Advancement of Technology through the International Cooperative R\&D program (Project No. P0016150).

\section{REFERENCES}

[1] A. Arıs, S. F. Oktug, and T. Voigt, "Security of internet of things for a reliable internet of services," Autonomous Control for a Reliable Internet of Services, 2018.

[2] E. Boo, S. Raza, J. Hoglund, and J. Ko, "Fdtls: Supporting dtls-based combined storage and communication security for iot devices," in 2019 IEEE 16th International Conference on Mobile Ad Hoc and Sensor Systems (MASS), pp. 127-135, 2019.

[3] H. Kwon, J. Ahn, and J. Ko, "Lightcert: On designing a lighter certificate for resource-limited internet-of-things devices," Transactions on Emerging Telecommunications Technologies, vol. 30, no. 10, p. e3740, 2019. e3740 ett.3740.

[4] I. Butun, S. D. Morgera, and R. Sankar, "A survey of intrusion detection systems in wireless sensor networks," IEEE communications surveys \& tutorials, vol. 16, no. 1, pp. 266-282, 2013.

[5] S. Raza, L. Wallgren, and T. Voigt, "Svelte: Real-time intrusion detection in the internet of things," Ad hoc networks, vol. 11, no. 8, pp. 26612674, 2013

[6] C.-F. Tsai, Y.-F. Hsu, C.-Y. Lin, and W.-Y. Lin, "Intrusion detection by machine learning: A review," expert systems with applications, vol. 36 , no. 10, pp. 11994-12000, 2009.

\footnotetext{
${ }^{1}$ The target is to provide the Cooja changes to upstream Cooja but a preliminary version of Multi-Trace is available at https:/github.com/nfi/multitrace
} 
[7] S. K. Wagh, V. K. Pachghare, and S. R. Kolhe, "Survey on intrusion detection system using machine learning techniques," International Journal of Computer Applications, vol. 78, no. 16, 2013.

[8] S. Revathi and A. Malathi, "A detailed analysis on nsl-kdd dataset using various machine learning techniques for intrusion detection," International Journal of Engineering Research \& Technology (IJERT), vol. 2, no. 12, pp. 1848-1853, 2013.

[9] F. Österlind, A. Dunkels, J. Eriksson, N. Finne, and T. Voigt, "Crosslevel sensor network simulation with cooja," in Proceedings of the First IEEE International Workshop on Practical Issues in Building Sensor Network Applications (SenseApp 2006), (Tampa, Florida, USA), Nov. 2006.

[10] I. Essop, J. C. Ribeiro, M. Papaioannou, G. Zachos, G. Mantas, and J. Rodriguez, "Generating datasets for anomaly-based intrusion detection systems in iot and industrial iot networks," Sensors, vol. 21, no. 4, p. $1528,2021$.

[11] A. Dunkels, B. Grönvall, and T. Voigt, "Contiki - a lightweight and flexible operating system for tiny networked sensors," in Proceedings of the IEEE Workshop on Embedded Networked Sensor Systems (IEEE Emnets), (Tampa, Florida, USA), Nov. 2004.

[12] J. Eriksson, F. Österlind, N. Finne, N. Tsiftes, A. Dunkels, T. Voigt, R. Sauter, and P. J. Marrón, "Cooja/mspsim: Interoperability testing for wireless sensor networks," in SIMUTools 2009, (Rome, Italy), Mar. 2009.

[13] J. Ko, J. Eriksson, N. Tsiftes, S. Dawson-Haggerty, M. Durvy, J. Vasseur A. Terzis, A. Dunkels, and D. Culler, "Beyond Interoperability: Pushing the Performance of Sensornet IP Stacks," in Proceedings of the International Conference on Embedded Networked Sensor Systems (ACM SenSys), (Seattle, WA, USA), Nov. 2011.

[14] J. Eriksson, A. Dunkels, N. Finne, F. Österlind, and T. Voigt, "Mspsim - an extensible simulator for msp430-equipped sensor boards," in Proceedings of the European Conference on Wireless Sensor Networks (EWSN), (Delft, The Netherlands), Jan. 2007.

[15] T. Winter (Ed.), P. Thubert (Ed.), and RPL Author Team, "RPL: IPv6 Routing Protocol for Low power and Lossy Networks," Mar. 2012. RFC 6550 .

[16] T. Watteyne, M.-R. Palattella, and L. A. Grieco, "Using ieee 802.15 . 4e time-slotted channel hopping (tsch) in the internet of things (iot): Problem statement," Internet Engineering Task Force, 2015.

[17] A. Dunkels, F. Österlind, N. Tsiftes, and Z. He, "Software-based online energy estimation for sensor nodes," in Proceedings of the IEEE Workshop on Embedded Networked Sensor Systems (IEEE Emnets), (Cork, Ireland), June 2007.

[18] A. Dunkels, J. Eriksson, N. Finne, and N. Tsiftes, "Powertrace: Networklevel power profiling for low-power wireless networks," Tech. Rep. T2011:05, Swedish Institute of Computer Science, Mar. 2011.

[19] J. Huang, C. Samplawski, D. Ganesan, B. Marlin, and H. Kwon, "Clio: enabling automatic compilation of deep learning pipelines across iot and cloud," in Proceedings of the 26th Annual International Conference on Mobile Computing and Networking, pp. 1-12, 2020.

[20] S. McCanne, "libpcap: An architecture and optimization methodology for packet capture," 2011

[21] J. Kovač, J. Crnogorac, E. Kočan, and M. Vučinić, "Sniffing multi-hop multi-channel wireless sensor networks," pp. 1-4, 2020.

[22] C. Kolias, G. Kambourakis, A. Stavrou, and S. Gritzalis, "Intrusion detection in 802.11 networks: Empirical evaluation of threats and a public dataset," IEEE Communications Surveys Tutorials, vol. 18, no. 1, pp. 184-208, 2016.

[23] X. Vilajosana, T. Watteyne, T. Chang, M. Vučinić, S. Duquennoy, and P. Thubert, "Ietf 6tisch: A tutorial," IEEE Communications Surveys \& Tutorials, vol. 22, no. 1, pp. 595-615, 2019.

[24] X. Fafoutis, L. Marchegiani, A. Elsts, J. Pope, R. Piechocki, and I. Craddock, "Extending the battery lifetime of wearable sensors with embedded machine learning," pp. 269-274, 2018.

[25] A. Shoeb, D. Carlson, E. Panken, and T. Denison, "A micropower support vector machine based seizure detection architecture for embedded medical devices," pp. 4202-4205, 2009.

[26] M. Merenda, C. Porcaro, and D. Iero, "Edge machine learning for aienabled iot devices: a review," Sensors, vol. 20, no. 9, p. 2533, 2020.

[27] M. Sharma, H. Elmiligi, and F. Gebali, "A novel intrusion detection system for rpl-based cyber-physical systems," IEEE Canadian Journal of Electrical and Computer Engineering, 2021.
[28] P. Pongle and G. Chavan, "Real time intrusion and wormhole attack detection in internet of things," International Journal of Computer Applications, vol. 121, no. 9, 2015.

[29] M. N. Napiah, M. Y. I. B. Idris, R. Ramli, and I. Ahmedy, "Compression header analyzer intrusion detection system (cha-ids) for 6lowpan communication protocol," IEEE Access, vol. 6, pp. 16623-16638, 2018.

[30] Y. Al-Hadhrami and F. K. Hussain, "Real time dataset generation framework for intrusion detection systems in iot," Future Generation Computer Systems, vol. 108, pp. 414-423, 2020.

[31] A. Verma and V. Ranga, "Rpl-nidds17-a data set for intrusion detection in rpl based 6lowpan networks (internet of things)," Int. J. Grid Distrib. Comput., vol. 11, no. 8, pp. 43-56, 2018.

[32] A. Verma and V. Ranga, "Elnids: Ensemble learning based network intrusion detection system for rpl based internet of things," in 2019 4th International conference on Internet of Things: Smart innovation and usages (IoT-SIU), pp. 1-6, IEEE, 2019.

[33] H. Wang, L. Barriga, A. Vahidi, and S. Raza, "Machine learning for security at the iot edge-a feasibility study," in International Workshop on Machine Learning Security and Privacy: Experiences and Applications (WISECML'19), 2019.

[34] E. Kfoury, J. Saab, P. Younes, and R. Achkar, "A self organizing map intrusion detection system for rpl protocol attacks," International Journal of Interdisciplinary Telecommunications and Networking (IJITN), vol. 11, no. 1, pp. 30-43, 2019.

[35] M. Anagnostopoulos, G. Spathoulas, B. Viaño, and J. AugustoGonzalez, "Tracing your smart-home devices conversations: A real world iot traffic data-set," Sensors, vol. 20, no. 22, p. 6600, 2020.

[36] G. Suciu, M.-A. Sachian, I. Petre, D. Petrache, G. Petrescu, V. Mantzana, I. Gkotsis, F. Bertone, L. Viarengo, and S. Andronello, "Cyber-physical threat detection platform designed for healthcare systems," Annals of Disaster Risk Sciences: ADRS, vol. 3, no. 1, pp. 0-0, 2020. 